\title{
Inmunogenicidad de vacunas inactivadas de Salmonella sp de uso en la industria avícola argentina
}

\author{
Chacana, P.'; Leiva, C. ; Joaquim, P. ${ }^{1}$; Bombicino, M. ${ }^{2}$; Terrera, M.V. ${ }^{2}$ \\ ${ }^{1}$ Instituto Patobiología, CICVyA-INTA (Castelar, Buenos Aires, Argentina). \\ ${ }^{2}$ Departamento Aves, DILAB-SENASA (Martínez, Bs.Aires, Argentina). \\ E-mail: chacana.pablo@inta.gob.ar
}

\begin{abstract}
Resumen
Chacana, P.; Leiva, C.; Joaquim, P.; Bombicino, M.; Terrera, M.V.: Inmunogenicidad de vacunas inactivadas de Salmonella sp de uso en la industria avícola argentina. Rev. Vet. 31: 1, 69-73, 2020. La salmonelosis es una de las enfermedades infecciosas más difundidas a nivel mundial, que afecta tanto a seres humanos como a diferentes especies animales, estando históricamente vinculada a la avicultura. Salmonella sp es un microorganismo que puede transmitirse a través de los alimentos y ello implica su impacto en la salud pública, así como también en el comercio internacional de productos y subproductos avícolas. La vacunación es una de las herramientas fundamentales para la contención de estas bacterias y su efectividad requiere de controles de calidad para medir la inmunogenicidad de las mismas. El objetivo de este trabajo fue determinar la inmunogenicidad de tres vacunas comerciales de Salmonella $s p$ aviares de uso en la producción avícola argentina, mediante la inmunización grupal y la determinación de títulos séricos específicos. Grupos de aves fueron vacunadas según un procedimiento estandarizado que incluyó dos vacunaciones. Se observó diversidad de los títulos-aglutinación de Salmonella enteritidis entre las distintas vacunas analizadas, si bien todas ellas generaron seroconversión en las aves. La vacuna 1 presentó los mayores promedios de título, tanto en la primera como en la segunda vacunación, siendo seguida por la vacuna 3 y la vacuna 2 . El control de las vacunas tiene como objetivo final estimular la mejoría continua de su calidad para la producción de alimentos más inocuos para la población.
\end{abstract}

Palabras clave: gallinas, Salmonella $s p$, vacunas inactivadas, inmunogenicidad, control de calidad.

\begin{abstract}
Chacana, P.; Leiva, C.; Joaquim, P.; Bombicino, M.; Terrera, M.V.: Immunogenicity of inactivated Salmonella sp vaccines used in the poultry industry in Argentina. Rev. Vet. 31: 1, 69-73, 2020. Salmonellosis is one of the most widespread infectious diseases worldwide, affecting humans and several animal species, and it has been historically linked to poultry production. Salmonella $s p$ is a food-borne microorganism with impact on public health and also on the global trade of poultry products and by-products. Vaccination is one of the main tools to the pathogen and its effectiveness requires quality controls to measure their immunogenicity. The objective of this work was to determine the immunogenicity of three commercial avian Salmonella $s p$ vaccines used in Argentinean poultry production by immunizing groups of birds and determining specific serum titers. Groups of birds were vaccinated according to a standardized procedure that comprises two vaccinations. Diversity was observed in the agglutination titers against Salmonella enteritidis among the vaccines analyzed, although all of them generated seroconversion in the birds. The vaccine 1 presented the biggest title averages, as much in the first one as in the second vaccination, being continued by the vaccine 3 and the vaccine 2 . The final goal of the control of vaccines is to stimulate continuous improvement of their quality to produce safer food for the population.
\end{abstract}

Key words: poultry, Salmonella $s p$, inactivated vaccines, immunogenicity, quality control.

\section{INTRODUCCIÓN}

La salmonelosis es una de las enfermedades infecciosas más difundidas a nivel mundial, que afecta tanto

Recibido: julio 2019 / Aceptado: noviembre 2019 a seres humanos como a diferentes especies animales. De hecho, Salmonella $s p$ se encuentra a nivel mundial entre los principales agentes etiológicos vinculados con las zoonosis y la intoxicación alimentaria, conjuntamente con Campylobacter sp, generalmente asociada a alimentos que se consumen crudos. 
La avicultura es una de las producciones animales que históricamente ha estado vinculada a Salmonella $s p$ y la presencia de este germen patógeno en la producción tiene no sólo consecuencias en la sanidad animal, sino también en la salud pública e incluso en el comercio internacional de productos y subproductos avícolas.

Salmonella ser. gallinarum biovar gallinarum (SG) y Salmonella ser. gallinarum biovar pullorum (SP) son los agentes etiológicos de la pullorosis y la tifosis aviar, respectivamente. Estas biovariedades son comúnmente llamadas "salmonelas inmóviles" debido a que son las únicas salmonelas que carecen de flagelos y pueden causar grandes pérdidas económicas debido a la morbimortalidad que causan en los lotes afectados (Rev Med Vet 84: 14-20).

Sin embargo, las aves pueden ser portadoras de otras salmonelas, comúnmente llamadas paratifoideas o "salmonelas móviles", poseedoras de potencial zoonótico ${ }^{19}$. La supervivencia de Salmonella en el medio ambiente es muy amplia porque este microorganismo es capaz de crecer entre 7 y $45^{\circ} \mathrm{C}$, y sólo se destruye cuando se expone a $65^{\circ} \mathrm{C}$ durante 10 a 15 minutos. Puede sobrevivir a $\mathrm{pH}$ ácido en los alimentos congelados e inclusive puede ser viable durante varios años en el medio ambiente ${ }^{7}$.

La prevalencia de las distintas serovariedades de Salmonella en las aves de producción varía en los distintos países. Algunas serovariedades emergen en un país o región por un período de tiempo y luego pueden desaparecer, lo que indicaría que la aparición de las serovariedades es dinámica y por lo tanto se debe realizar un monitoreo constante para contar con datos actualizados sobre la situación epidemiológica local y regional.

Durante mucho tiempo, S. ser. Typhimurium (ST) ha sido la serovariedad de mayor prevalencia, tanto en seres humanos como en la avicultura. Sin embargo, durante la década del 1980, la prevalencia de S. ser. Enteritidis (SE) fue incrementándose, superando en la actualidad a $\mathrm{ST}^{12}$. Tradicionalmente, SE es la serovariedad que más frecuentemente se ha aislado en América Latina a partir de seres humanos, animales, alimentos $\mathrm{y}$ en el medio ambiente. En los últimos años otras serovariedades han comenzado a prevalecer en la avicultura. Según estudios realizados recientemente, $S$. ser. Heidelberg es la serovariedad de mayor prevalencia en granjas de pollos de engorde en Argentina y Brasil 18, 22 .

En el caso de Argentina, durante el período de octubre de 2009 a octubre de 2011 se realizó un estudio de prevalencia de Salmonella en granjas comerciales, llevado a cabo por un programa de aves y granja de la Dirección Nacional de Sanidad Animal (SENASA), enfocado al aislamiento de serovariedades móviles en granjas de pollos de engorde y gallinas ponedoras, siguiendo las normativas de la Unión Europea.

La bacteria fue detectada en el $45 \%$ de las granjas de pollos de engorde y las cepas aisladas correspondieron a 36 serovariedades diferentes. Las más frecuentes fueron Heidelberg (21,79\%), Thompson (13,73\%) y
Schwarzengrund (11.04\%); SE y ST fueron aisladas en 7 y 8 casos respectivamente $(2.09 \%)$.

A partir del año 2016 el SENASA desarrolló el "Programa de vigilancia y control de la contaminación por Salmonella sp en granjas avícolas comerciales" (Resolución 86/2016), que tiene como objetivo disminuir la prevalencia del germen en la avicultura industrial argentina. El mismo complementa el "Programa de control de mycoplasmosis y salmonelosis de las aves, prevención y vigilancia de enfermedades exóticas y de alto riesgo en planteles de reproducción" que fue creado en el año 2002.

Entre las estrategias para el control de salmonelosis, la bioseguridad en granjas reviste la mayor importancia y está contemplada en la resolución del SENASA Nro. 542/2010 y su complementaria No 106/2013, siendo la vacunación una valiosa herramienta para el control de la enfermedad. Existen diferentes tipos de vacunas contra Salmonella, a saber: inactivadas, vivas atenuadas y a subunidad.

Se han publicado diversas investigaciones sobre el desarrollo y eficacia de vacunas para el control de las salmonelas en las aves, que exploran diferentes enfoques ${ }^{13,23}$, pero pocas de ellas están disponibles comercialmente y por lo tanto existe la demanda mundial constante de nuevas vacunas seguras y definidas ${ }^{6}$.

Entre las vacunas vivas disponibles en el mercado, la cepa 9R de SG ha sido utilizada principalmente para el control de la tifosis aviar y su aplicación está dirigida a gallinas de postura comercial. Se ha demostrado que esta vacuna también presenta protección cruzada contra SE. Por otro lado, también se han desarrollado comercialmente vacunas vivas que incorporan cepas atenuadas de SE y ST ${ }^{8}$ y pueden ser administradas a gallinas ponedoras y reproductores.

Algunas de estas vacunas han demostrado su capacidad de proteger contra otras serovariedades distintas a las incluidas en su formulación (Avian Dis 50: 280 283). Diferente es el caso de las vacunas inactivadas de tipo bacterina, donde existe mayor oferta comercial por parte de distintos laboratorios productores, tanto nacionales como internacionales. La mayoría de estas vacunas inactivadas incorporan SE y ST en su formulación y pueden también incluir otras serovariedades, dependiendo de la prevalencia del patógeno en las distintas regiones donde son comercializadas y aplicadas.

En general, tales vacunas están destinadas a aves reproductoras y gallinas ponedoras, teniendo como objetivo otorgar inmunidad materna, disminuir el riesgo de contaminación en huevos de consumo y bajar los niveles microbianos del tracto digestivo de las aves.

Estas bacterinas usualmente se encuentran formuladas tanto con adyuvantes oleosos (generalmente aceite en agua) o acuosos (gel de hidróxido de aluminio) y estimulan la producción de altos niveles de anticuerpos circulantes que, dependiendo de la calidad de la vacuna, pueden persistir durante todo el período productivo ${ }^{17}$.

Generalmente, la eficacia de las vacunas es evaluada según su capacidad de disminuir los niveles de colo- 
nización intestinal y sistémica, así como de reducir la tasa de morbilidad-mortalidad en modelos de infección experimental o en ensayos llevados a cabo en condiciones productivas. Los niveles de protección dependen de muchos factores, tales como la vía de administración, la edad y línea genética de las aves y -en gran medidade la cepa de Salmonella y la dosis o niveles de desafío.

Por esta razón, siempre ha sido muy difícil comparar las vacunas disponibles respecto a su eficacia. La efectividad de las vacunas inactivadas requiere de controles de calidad para medir la inmunogenicidad de las mismas.

Si bien existen distintas técnicas que se pueden aplicar para evaluar la serología contra este patógeno, tales como ELISA ${ }^{15}$ y aglutinación en placa, la metodología de aglutinación lenta en microplacas viene siendo utilizada desde hace varios años en distintas investigaciones, muy probablemente debido a su simplicidad y versatilidad ${ }^{9}$.

En este contexto, en la Argentina se ha establecido un procedimiento para determinar la inmunogenicidad de las vacunas inactivadas de Salmonella mediante la técnica de aglutinación lenta en placa, una de las recomendadas por la OIE ${ }^{16}$.

El objetivo de este trabajo fue determinar la inmunogenicidad de tres vacunas contra Salmonella, de uso en la avicultura argentina.

\section{MATERIAL Y MÉTODOS}

Aves. Se utilizaron aves ponedoras (híbridos comerciales) de 4 semanas de vida, derivadas de la raza Leghorn. Las mismas recibieron agua y alimento ad libitum durante todo el ensayo, según los requerimientos de la línea utilizada. A lo largo del tiempo que duraron los ensayos se verificó el status libre de Salmonella $s p$ mediante hisopado cloacal y cultivo bacteriológico.

Vacunas y vacunación. Se analizaron tres vacunas inactivadas comerciales, del tipo bacterina, nacionales e importadas, de uso en la Argentina, que estaban formuladas con distintas serovariedades de Salmonella $s p$. Todas ellas incluían SE en su formulación. Las aves fueron vacunadas por vía subcutánea en la zona dorsal del cuello con una dosis de cada vacuna, de acuerdo con las instrucciones del laboratorio elaborador.

Grupos experimentales. Para cada uno de los ensayos, las aves fueron divididas en dos grupos: a) grupo vacunado, conformado por 25 aves que recibieron la primera dosis de vacuna durante la semana 4 de vida y la segunda dosis durante la semana 8 de vida; y b) grupo control, que incluyó 10 aves que no fueron inmunizadas.

Toma de muestras. Se tomaron muestras individuales de sangre de todas las aves antes de la primera vacunación (suero pre-inmune) y a las 4 semanas luego de la primera y segunda inmunización (sueros post- $1^{\circ} \mathrm{y}$ post- $2^{\circ}$ inmunización, respectivamente). La sangre fue extraída a partir de la vena alar y los sueros se conservaron a $-20^{\circ} \mathrm{C}$ hasta su utilización. Las aves fueron sacrificadas mediante dislocación cervical seguida de exsanguinación en el momento de la última toma de muestras, a las 12 semanas de vida.

Análisis serológico. El título de anticuerpos presentes en las muestras de suero fue determinado mediante aglutinación lenta en placas de 96 pocillos con fondo en "U". Debido a la variabilidad en la formulación antigénica de las vacunas respecto a las serovariedades de Salmonella incluidas, se determinaron los títulos de aglutinación para SE, serotipo que formaba parte de la formulación de todas ellas. Para esto, una suspensión de SE fue inactivada con formalina y su concentración fue ajustada al tubo 1 de la escala nefelométrica de McFarland (correspondiente a alrededor de $1 \times 10^{8}$ bacterias $/ \mathrm{ml}$ ). Se realizaron diluciones iniciales 1:10 de los sueros y posteriormente diluciones seriadas binarias (entre 1:20 y 1:5120) que fueron enfrentadas con la suspensión del antígeno. Asimismo, todas las placas incluyeron controles negativos (antígeno enfrentado a solución fisiológica) y controles positivos (suero de aves inmunizadas con SE de título previamente determinado). Las placas fueron incubadas a $37^{\circ} \mathrm{C} \mathrm{du}-$ rante $18 \mathrm{~h}$ y posteriormente se llevó a cabo la lectura de las mismas. El título de aglutinación fue determinado como la mayor dilución de los sueros que presentó una reacción positiva en la prueba.

Análisis estadístico. La diferencia de medias entre los distintos tiempos de toma de muestras para cada una de las vacunas fue analizada mediante ANOVA seguido del test de comparación múltiple de medias de Tukey con un nivel de significancia de 0,05.

\section{RESULTADOS}

Todas las aves permanecieron negativas a Salmonella durante todo el período de los ensayos. Asimismo, todas las aves del grupo control presentaron títulos de aglutinación menores a 1:20 en todas las muestras analizadas.

Se observaron diferencias respecto a la seroconversión de las aves vacunadas con las distintos inmunógenos analizados. En la Tabla 1 se muestra la media

Tabla 1. Ttítulos de aglutinación lenta en microplaca contra Salmonella ser. enteritidis de aves inmunizadas con las diferentes vacunas analizadas.

\begin{tabular}{cccc}
\hline \multirow{2}{*}{ vacuna } & \multicolumn{3}{c}{ media geométrica del título de aglutinación } \\
\cline { 2 - 4 } & pre-inmune & post- $^{\mathrm{o}}$ inmuniz. & post- $^{\mathrm{o}}$ inmuniz. $^{\mathrm{in}}$ \\
\hline 1 & $<20$ & $999,3(201,2)^{\mathrm{a}}$ & $1698(214,4)^{\mathrm{c}}$ \\
2 & $<20$ & $150,2(60)^{\mathrm{a}}$ & $502,1(164,2)^{\mathrm{b}}$ \\
3 & $<20$ & $286,8(155)^{\mathrm{a}}$ & $815,7(150)^{\mathrm{b}}$ \\
\hline
\end{tabular}

Letras diferentes indican diferencias significativas $(p<0,05)$ entre cada uno de momentos de toma de muestras para cada una de las vacunas. 
geométrica de los títulos serológicos contra SE determinados en cada una de las muestras analizadas de las aves vacunadas. La vacuna 1 presentó los mayores valores promedios de títulos tanto luego de la primera como de la segunda vacunación, seguida por la vacuna 3 y la vacuna 2 .

\section{DISCUSIÓN}

La metodología utilizada fue eficaz para determinar diferencias en los niveles de seroconversión después de las vacunaciones. En todos los casos, las aves que se mantuvieron sin vacunar, así como las muestras de sueros tomadas antes de las vacunaciones, presentaron títulos contra SE menores a los detectables según las diluciones ensayadas, lo que indica que no existió exposición a antígenos de Salmonella ambientales durante todo el período del ensayo.

Por lo tanto, los valores serológicos determinados en las aves vacunadas serían consecuencia solamente de la administración de los inmunógenos y no hubo interferencia con otros antígenos que pudieran estar presentes durante los ensayos.

La técnica de aglutinación lenta en microplaca, si bien presenta menor sensibilidad que otras, tiene algunas ventajas en su implementación dentro del control de calidad de vacunas debido a su simplicidad en la ejecución, bajos costos relativos y versatilidad respecto a los antígenos que pueden utilizarse.

Ello permite que pueda ser llevada a cabo en laboratorios de baja complejidad y consecuentemente la implementación masiva de la misma. Se observó diversidad entre las distintas vacunas analizadas respecto a su capacidad inmunogénica en el procedimiento utilizado. Entre las distintas vacunas, y luego de la segunda inmunización, los títulos de aglutinación promedio variaron entre 1:502,1 y 1:1698.

Estas diferencias posiblemente se deben a las características de producción y concentración de los antígenos utilizados en las formulaciones, así como también a los adyuvantes incluidos en las mismas. En todos los casos, se observaron diferencias significativas respecto a los títulos determinados luego de la primera y segunda vacunación, lo que indica que las vacunas analizadas generaron respuesta contra SE y que la segunda vacunación tuvo la capacidad de incrementar significativamente los títulos específicos.

Si se considera que uno de los criterios de aprobación de vacunas es el incremento significativo entre los títulos posteriores a la primera y segunda vacunación, las tres vacunas fueron satisfactorias, si bien la capacidad inmunogénica de la vacuna 1 fue superior. La diversidad de productos disponibles en el mercado ofrece distintos recursos y herramientas para complementar el control de Salmonella en la avicultura.

Las vacunas vivas, si son administradas en forma oral, han demostrado ser eficaces para disminuir la colonización de las aves en sus primeras etapas de vida. Sin embargo, existen autores que consideran que las vacunas inactivadas o a subunidad presentan mayor seguridad tanto para el ave como para el consumidor. Por esta razón los laboratorios continúan desarrollando nuevos adyuvantes que puedan estimular en forma más eficiente al sistema inmune mediante el uso de vacunas inactivadas.

Es importante considerar que los programas de control del microorganismo en las granjas deben basarse en buenas prácticas de manejo, que incluyan estrictas medidas sanitarias y de bioseguridad. La vacunación es una de las herramientas disponibles pero por sí misma no solucionará el problema, si bien contribuye a la prevención y reducción de los niveles ambientales de Salmonella. Asimismo, en el contexto mundial de reducción del uso de antibióticos, las vacunas son consideradas alternativas para mejorar la sanidad en la producción animal ${ }^{11,14}$.

El control oficial estimula la mejoría continua de la calidad de las vacunas que utiliza el sector avícola, cuyo objetivo final consiste en la producción de alimentos más seguros para la población.

Agradecimientos. Los autores agradecen la colaboración de la Dra. Analia Kaloghlian y de los técnicos Damián Fuente y Jesica Bucci. Este trabajo fue llevado a cabo en el marco del Convenio de Cooperación Técnica INTA-Senasa. denominado "Salmonella y Campylobacter de importancia en avicultura".

\section{REFERENCIAS}

1. Andres VM, Davies RH. 2015. Biosecurity measures to control Salmonella and other infectious agents in pig farms. Compr Rev Food Sci \& Food Saf 14: 317-335.

2. Barrow P. 1994. Serological diagnosis of Salmonella serotype enteritidis infections in poultry by ELISA and other tests. Int J Food Microbiol 21: 55-68.

3. Barrow P. 2007. Salmonella infections: immune and nonimmune protection with vaccines. Avian Pathol 36: 1-13.

4. Barrow P, Lovell M, Berchieri A. 1990. Immunization of laying hens against Salmonella enteritidis with live attenuated vaccines. Vet Rec 126: 241-242.

5. Caria D. 2017. Salmonelosis en granjas de pollos de engorde en la República Argentina. Tesis de Maestría, Universidad de Buenos Aires, Argentina, p. 16-28.

6. Desin T, KösterW, Potter A. 2013. Salmonella vaccines in poultry: past, present and future. Expert Rev Vaccines 12: 87-96.

7. Finn S et al. 2013. Mechanisms of survival, responses, and sources of Salmonella in low-moisture environments. Front Microbiol 4: 331.

8. Gantois I et al. 2006. Oral immunization of laying hens with the live vaccine strains of TAD Salmonella vac E and TAD Salmonella vac T reduces internal egg contamination with Salmonella enteritidis. Vaccine 24: 6250-6255.

9. Gast R, Beard C. 1990. Serological detection of experimental Salmonella enteritidis infections in laying hens. Avian Dis 34: 721-728. 
10. Heredia N, García S. 2018. Animals as sources of foodborne pathogens: A review. Anim Nutr 4: 250-255.

11. Hoelzer K et al. 2018. Vaccines as alternatives to antibiotics for food producing animals. Vet Res 49: 64-70.

12. Humphrey T, Jorgensen, F. 2006. Pathogens on meat and infection in animals. Establishing a relationship using Campylobacter and Salmonella as example. Meat Science 74: 89-97.

13. Matulova M et al. 2013. SPI-1 defective mutants of Salmonella enterica induce cross-protective immunity in chickens against challenge with serovars Typhimurium and Enteritidis. Vaccine 31: 3156-3162.

14. Nandre R, Dajeong L. 2015. Cross-protection against Salmonella typhimurium infection conferred by a live attenuated Salmonella enteritidis vaccine. Can J Vet Res 79: 16-21.

15. Nicholas R, Cullen G. 1991. Development and application of an ELISA for detecting antibodies to Salmonella enteritidis in chicken flocks. Vet Rec 128: 74-76.

16. OIE. 2018. Salmonelosis. Manual de pruebas de diagnóstico y de vacunas para animales terrestres (versión online), capítulo 2.9.8, pag. 1-18.
17. Paiva J et al. 2009. Efficacy of several Salmonella vaccination programs against experimental challenge with $S$ gallinarum in commercial brown layer and broiler breeder hens. Rev Bras Ciência Avíc 11: 65-72.

18. Pulido LM, Sánchez IR, Guard J, Pinheiro NV. 2013. Assignment of serotype to Salmonella enterica isolates obtained from poultry and their environment in southern Brazil. Lett Appl Microbiol 57: 288-294.

19. Revolledo L, Ferreira A. 2012. Current perspectives in avian salmonellosis: vaccines and immune mechanisms of protection. J Appl Poult Res 21: 418-431

20. Rios A et al. 2016. Alternatives to overcoming bacterial resistances: state-of-the-art. Microbiol Res 191: 51-80.

21. Vandeplas $\mathbf{S}$ et al. 2010. Salmonella in chicken: Current and developing strategies to reduce contamination at farm level. J Food Prot 73: 774-785.

22. Vossrech D et al. 2015. A temporal study of Salmonella enterica serotypes from broiler farms in Brazil. Poult Sci 94: 433-441.

23. Yang Y et al. 2017. Evaluation of recombinant Salmonella vaccines to provide cross-serovar and cross-serogroup protection. Poult Sci 96: 4352-4360. 